\title{
Upper airway collapse and reopening induce inflammation in a sleep
} apnoea model

\author{
I. Almendros*, A. Carreras*, J. Ramírez ${ }^{\#}$, J.M. Montserrat ${ }^{\uparrow,+}$, \\ D. Navajas ${ }^{\star,+,}$ and R. Farré ${ }^{\star,+}$
}

ABSTRACT: The upper airway of obstructive sleep apnoea patients is subjected to recurrent negative pressure swings promoting its collapse and reopening. The aim of the present study was to ascertain whether this mechanical stress induces upper airway inflammation in a rat model.

The upper airway of Sprague-Dawley rats was subjected to a periodic pattern of recurrent negative $\left(-40 \mathrm{cmH}_{2} \mathrm{O}, 1 \mathrm{~s}\right)$ and positive $\left(4 \mathrm{cmH}_{2} \mathrm{O}, 2 \mathrm{~s}\right)$ pressures inducing collapse and reopening for $5 \mathrm{~h}$. Rats that were instrumented but not subjected to negative pressure swings were used as controls. The gene expression of the pro-inflammatory biomarkers macrophage inflammatory protein (MIP)-2, tumour necrosis factor (TNF)- $\alpha$, interleukin (IL)-1 $\beta$ and P-selectin in the soft palate and larynx tissues was assessed by real-time PCR.

A marked overexpression of MIP-2, TNF- $\alpha$, IL-1 $\beta$ and P-selectin ( 40-, 24-, 47- and 7-fold greater than controls, respectively) was observed in the larynx tissue; similar results were found in the soft palate tissue ( 14-, 7-, 35- and 11-fold greater than controls, respectively).

Recurrent upper airway collapse and reopening mimicking those experienced by obstructive sleep apnoea patients triggered an early local inflammatory process. These results could explain the inflammation observed in the upper airway of obstructive sleep apnoea patients.

KEYWORDS: Airway collapse, airway reopening, inflammation, negative pressure, obstructive sleep apnoea, upper airway

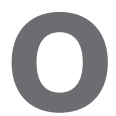

bstructive sleep apnoea (OSA) syndrome is a prevalent disorder characterised by recurrent upper airway obstructions with associated oxygen desaturation and disruption of sleep architecture. These recurrent obstructions and the associated breathing disturbances are caused by an increased collapsibility of the upper airway in OSA patients. Specifically, the negative pressure, normally associated with the beginning of inspiration, promotes a reduction in the lumen of the abnormally compliant upper airway with a consequent increase in airflow resistance. Sustaining inspiration through an airway with increased resistance requires a more negative inspiratory pressure which, in turn, contributes to a progressive obstruction of the upper airway and finally to the obstructive apnoea [1, 2]. The airway closure is resolved with the activation of the airway dilatory muscles caused by the patient's arousal.

As a consequence of the repetitive events of negative intraluminal pressure, collapse and

For editorial comments see page 255 . reopening, the upper airway exhibits structural and functional alterations such as inflammation and oedema in the lamina propria of the uvula mucosa, in the soft palate and in the upper airway muscle [3-5]. This mechanical challenge also causes upper airway sensorial dysfunction to stimuli such as temperature, pressure and vibration [6-8]. Given that the upper airway plays a crucial role in the pathophysiology of OSA, a detailed understanding of its potential inflammatory mechanisms is of major interest. Indeed, the inflammation of the soft-tissue structures in the pharynx could develop pharyngeal wall thickening, resulting in upper airway narrowing $[3,9]$, and could also promote neuromuscular dysfunction, with the subsequent reduction in the upper airway dilator tone [5]. The loss of sensitivity in the pharyngeal wall could impair upper airway reflex to intraluminal inspiratory negative pressure, increasing its collapsibility and, hence, exacerbating the apnoeic events.

Although it has been hypothesised that the events of negative pressure, collapse and reopening induce the inflammation resulting in upper airway

\section{AFFILIATIONS}

*Unitat de Biofísica i Bioenginyeria, Facultat de Medicina, Universitat de Barcelona, Institut d'Investigacions Biomèdiques Augustí Pi i Sunyer (IDIBAPS),

\#Dept Anatomia Patològica, Hospital Clínic,

- Servei Pneumologia, Hospital

Clínic,

${ }^{\S}$ Institut de Bioenginyeria de

Catalunya, Barcelona, and

${ }^{+}$Centro Investigación Biomédica en Red de Enfermedades Respiratorias

Bunyola, Spain

\section{CORRESPONDENCE}

R. Farré

Unitat de Biofísica i Bioenginyeria

Facultat Medicina

Casanova 143

08036 Barcelona

Spain

Fax: 34934035278

E-mail: rfarre@ub.edu

Received:

November 302007

Accepted after revision:

April 162008

\section{SUPPORT STATEMENT}

This work was supported in part by the Ministerio de Ciencia y

Tecnología (SAF2005-00110 and SAF2004-00684) and the Ministerio de Sanidad y Consumo (FISPI040929).

STATEMENT OF INTEREST None declared. 
dysfunction $[3,5]$, there is no evidence that this mechanical stress actually causes local inflammation. In fact, the well documented data obtained from patient studies [3-5] are inconclusive because different confounding pro-inflammatory stimuli (hypoxia, metabolic syndrome, obesity, etc.) may contribute to the upper airway changes described in OSA patients. Accordingly, the aim of the present study was to establish a realistic and well-controlled rat model in order to ascertain whether a collapse-reopening stimulus similar to that experienced by patients with OSA, is able to trigger an inflammatory response in the upper airway tissue.

\section{METHODS}

\section{Animal preparation}

The present investigation was carried out in 28 male SpragueDawley rats (body mass 250-300 g) and approved by the Ethics Committee for Animal Experimentation of the University of Barcelona (Barcelona, Spain). The animals were anesthaetised with $10 \%$ urethane $\left(1 \mathrm{~g} \cdot \mathrm{kg}^{-1}\right)$ and subjected to a double intubation at the tracheal level. A canula was inserted into the lower trachea and directed towards the lungs to allow the animal to breathe spontaneously. Another canula was inserted into the upper trachea and directed towards the upper airway to apply the recurrent negative pressure stimulus. A customised mask, open to the atmosphere through a solenoid electrovalve (L178B1; Sirai, Bussero, Italy), was tightly adjusted to the rat nose (fig. 1).

\section{Experimental setup}

The experimental setting employed to apply recurrent negative pressure swings to the upper airway is shown in figure 1 . The ports of a three-way solenoid electrovalve (L377B03G; Sirai) were connected to: the upper airway canula; a $-40 \mathrm{cmH}_{2} \mathrm{O}$ vacuum source; and a $4 \mathrm{cmH}_{2} \mathrm{O}$ positive-pressure source. By electronically controlling the valves, the upper airway was subjected to a periodic pattern of collapse (1 s) and reopening (2 s). Upper airway collapse was induced by simultaneously occluding the nasal mask valve and connecting the upper airway canula to the negative-pressure source (fig. 1a). Upper airway reopening was achieved by simultaneously opening the nasal mask to the atmosphere and connecting the upper airway canula to the positive-pressure source (fig. 1b). The temperature and humidity of the air in the positive-pressure source were kept under physiological conditions thereby avoiding drying of upper airway mucosa. Pressures at the nasal mask and at the upper airway canula were continuously measured (Microswitch 176PC28HD2; Honeywell, Scarborough, ON, Canada). Airflow at the trachea was measured with a pneumotachograph (Fleisch-000; Metabo, Epalinges, Switzerland) placed between the upper airway canula and the pressure source (not shown).

Figure 2 illustrates how the experimental setting (fig. 1) allowed the induction of upper airway collapse and reopening. The magnitude of the negative tracheal pressure swings applied was progressively reduced (six cycles) and then increased (four cycles). When tracheal pressure was higher than the intraluminal pressure causing collapse (critical

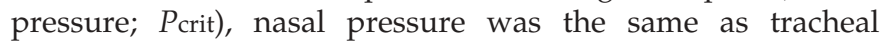
pressure, indicating that the upper airway remained open because the pressure applied to the trachea was transmitted to the nose. However, when tracheal pressure was lower than $P$ crit, nasal pressure was no longer reduced because the upper airway was collapsed and hence the negative pressure applied to the trachea could not be transmitted to the nose.

\section{Experimental protocol}

A total of 14 rats were subjected to a recurrent pattern of collapse and reopening for $5 \mathrm{~h}$ (fig. 3). In eight of these, the $P_{\text {crit }}$ of the upper airway was measured at the beginning and the end of application of the recurrent negative pressure swings. At the end of the experiment the rats were sacrificed. In these rats, the soft palate and larynx tissues were excised [10], frozen and stored at $-80^{\circ} \mathrm{C}$ for further inflammatory gene expression analysis. In the other six rats, the upper airway was excised completely [10] and embedded in an optimal cutting temperature compound, frozen and stored at $-80^{\circ} \mathrm{C}$ for histopathological analysis. As a control, a group of 14 rats were subjected to the same instrumentation and protocol with the exception that the recurrent negative pressure swings were not applied (by not connecting pressure sources; fig. 1).

\section{Gene expression analysis}

The tissue samples were defrosted and subsequently disrupted by means of a homogeniser (Polytron 2100; Kinematica, Luzern, Switzerland). The total RNA content of each sample was isolated using the 6100 Nucleic Acid PrepStation system (Applied Biosystems, Foster City, CA, USA) and the synthesis of cDNA

a)

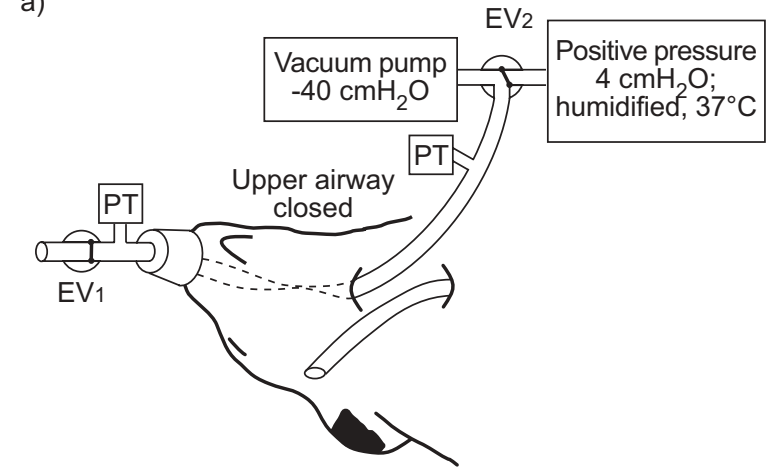

b)

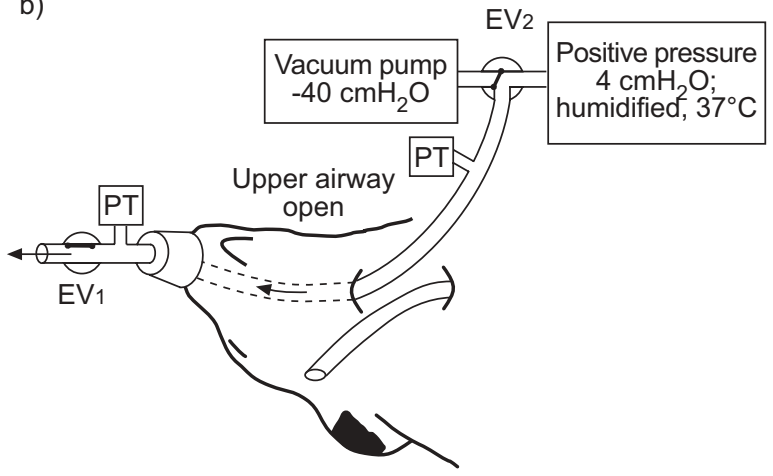

FIGURE 1. Schematic diagram of the experimental setup to apply an intermittent pattern of upper airway collapse (for $1 \mathrm{~s}$ ) and reopening (for $2 \mathrm{~s}$ ) in anesthaetised rats. a) Upper airway collapse was achieved by occluding the nasal mask and connecting the upper airway canula to a $-40 \mathrm{cmH}_{2} \mathrm{O}$ negative-pressure source. b) The upper airway was reopened by opening the nasal mask valve and connecting the upper airway canula to a $4 \mathrm{cmH}_{2} \mathrm{O}$ positive-pressure source. $\mathrm{EV} 1$ and $\mathrm{EV}_{2}$ : electrovalves 1 and 2, respectively; PT: pressure transducer. 


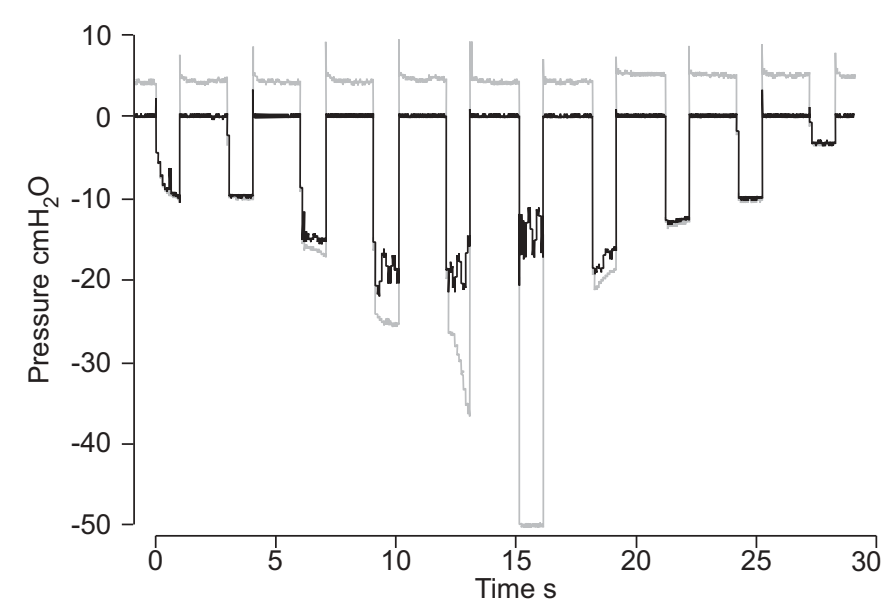

FIGURE 2. Example of the negative pressure applied intratracheally (grey line) and the pressure recorded at the nasal mask (black line). Both pressures were identical when tracheal pressure was higher than the critical pressure $\left(P_{\text {crit }}\right)$ of the upper airway $\left(\sim-15 \mathrm{cmH}_{2} \mathrm{O}\right.$ in this example). By contrast, when the negative pressure applied at the trachea was less than $P$ crit, nasal pressure was no longer reduced because the upper airway was collapsed and hence the negative pressure applied to the trachea could not be transmitted to the nose.

from total RNA was performed by High Capacity cDNA Kit reagents (Applied Biosystems). The amplification of cDNA was carried out, in triplicate, by real-time PCR (7300 RT-PCR; Applied Biosystems). Specific gene expression assays for macrophage inflammatory protein (MIP)-2, tumour necrosis factor (TNF)- $\alpha$, interleukin (IL)-1 $\beta$ and P-selectin (Rn00586403_m1, Rn00562055_m1, Rn00580432_m1 and Rn00565416_m1, respectively) were used (Assays on Demand ${ }^{\mathrm{TM}}$; Applied Biosystems). Gene expression of GAPD (Rn99999916_s1) was regarded as an endogenous reference for each rat. Finally, relative gene expression was quantified using the comparative method $2^{-\Delta \Delta \mathrm{Ct}}$ [11]. To this end, gene expression in the rats subjected to upper airway negative pressure was referenced to control rats.

\section{Upper airway histopathology}

A transversal section at the hypopharynx level was fixed in $4 \%$ formaldehyde for $48 \mathrm{~h}$ and paraffin-embedded. The paraffin blocks were cut in sections of $5 \mu \mathrm{m}$ and stained with haematoxylin and eosin. The degree of oedema and the presence of inflammatory cells in each sample were determined by two observers who did not know whether the preparation corresponded to rats subjected to the mechanical stimulus or to control rats.

\section{Statistical analysis}

Data are presented as mean \pm SEM. Comparisons between different groups were carried out by unpaired t-test or the Mann-Whitney rank sum test. The change in $P_{\text {crit }}$ of each rat induced by upper airway collapse/reopening was assessed by a paired t-test. A p-value $<0.05$ was considered statistically significant.

\section{RESULTS}

The 5-h pattern of recurrent collapse and reopening experienced by the upper airway induced an inflammatory process. A marked overexpression of the pro-inflammatory biomarkers
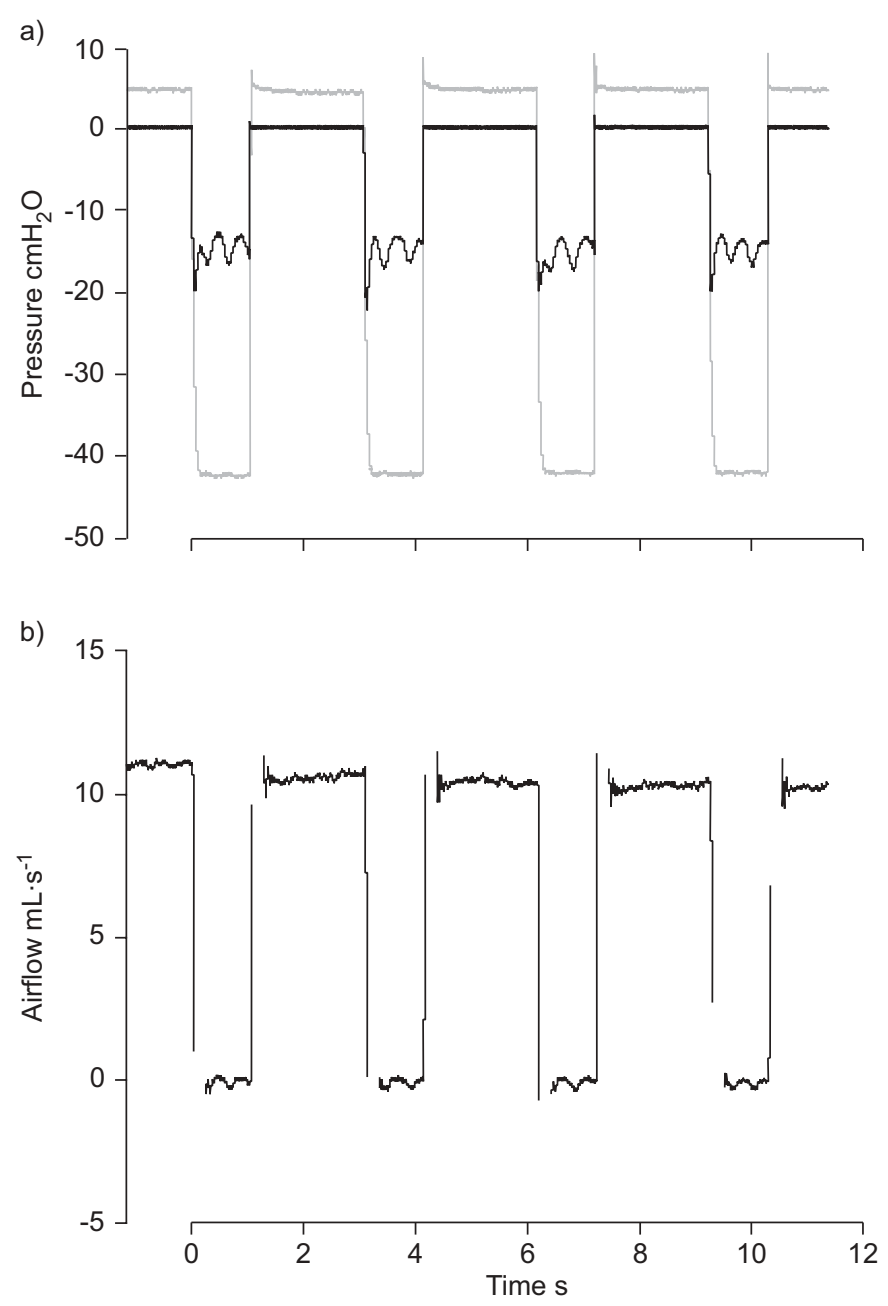

FIGURE 3. Example of the signals recorded during the 5-h application of intermittent upper airway collapse and reopening (data from the same rat used in fig. 2). a) Negative pressure swings applied intratracheally (grey line) and pressure recorded at the nasal mask (black line) and b) airflow recorded at the trachea. During the application of negative tracheal pressure, flow was nil indicating that the upper airway was collapsed. When positive pressure was applied at the trachea the flow signal indicated that the upper airway was open. The airflow signal around the transition between positive and negative pressure were not plotted to avoid the high-frequency noise induced by valve closing and opening.

MIP-2, TNF- $\alpha$, IL-1 $\beta$ and P-selectin ( 40-, 24-, 47- and 7-fold greater than controls, respectively) was observed in the larynx tissue (fig. 4); similar results were found in the soft palate tissue ( 14-, 7-, 35- and 11-fold greater than controls, respectively; fig. 5). By contrast, the histopathological analysis of the upper airway tissue showed no significant changes in oedema or in leukocyte infiltration when comparing the upper airway of rats subjected to collapse/reopening and controls (data not shown). On average, $P$ crit at the beginning of the experiment was $-19.4 \pm 1.6 \mathrm{cmH}_{2} \mathrm{O}$. No significant $(\mathrm{p}=0.11)$ change was observed after $5 \mathrm{~h}$ of upper airway mechanical challenge $\left(-15.7 \pm 2.3 \mathrm{cmH}_{2} \mathrm{O}\right)$.

\section{DISCUSSION}

An experimental setting specifically designed to apply negative pressure swings to the rat upper airway enabled the present 

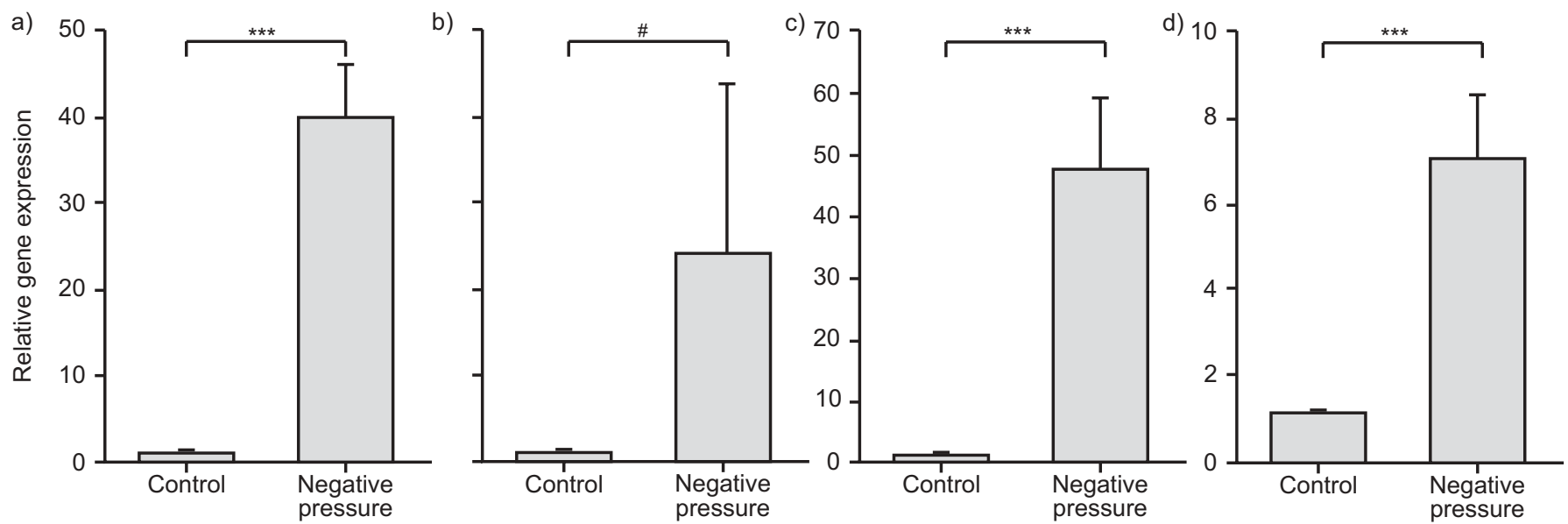

FIGURE 4. Relative gene expression of the pro-inflammatory biomarkers a) macrophage inflammatory protein-2, b) tumour necrosis factor- $\alpha, c)$ interleukin-1 $\beta$ and d) P-selectin in the larynx tissue of control rats and rats subjected to recurrent negative pressure swings in the upper airway. Data are presented as mean \pm SEM. ${ }^{\#}: p=0.002 ;{ }^{* \star *}: p<0.001$.

authors to induce a recurrent collapse/reopening pattern similar to the one experienced by the upper airway in patients with OSA. The magnitude of the negative pressure applied to the upper airway $\left(-40 \mathrm{cmH}_{2} \mathrm{O}\right)$ resembled the values of oesophageal pressures in OSA patients during obstructive events [2]. This mechanical stress was mild and short enough to avoid structural or functional effects in the upper airway tissue, as shown by the absence of histological differences between the rats subjected to the stimulus and the controls, and by the fact that $P$ crit did not change after the whole mechanical challenge. However, this realistic mechanical stimulus was able to trigger an early local inflammatory cascade. Indeed, a 5-h recurrent pattern of upper airway collapse and reopening caused by tracheal negative pressure swings resulted in a marked overexpression of the pro-inflammatory genes studied in the larynx and soft palate tissues.

a)

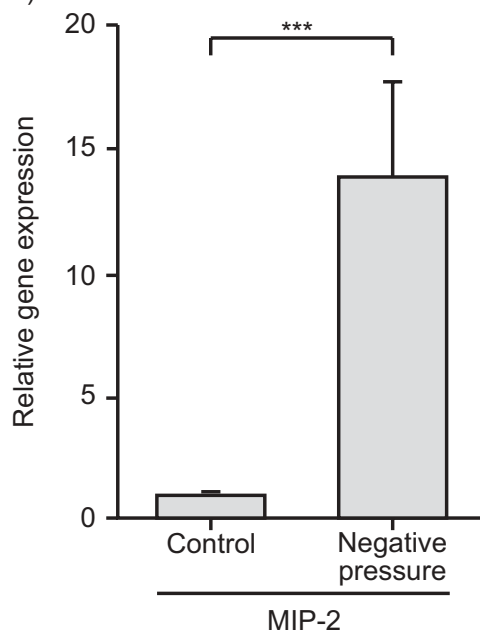

b)

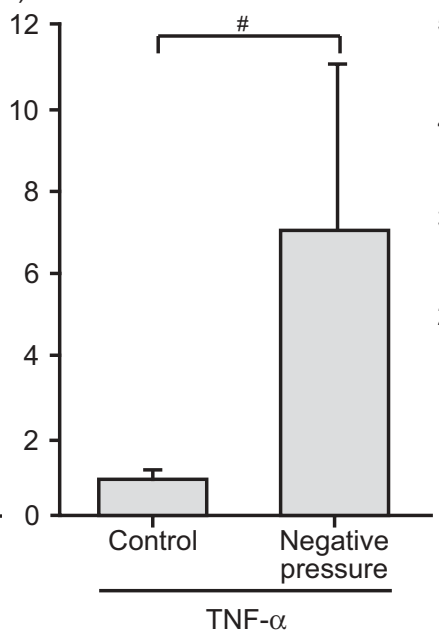

The in vivo isolated upper airway rat model has been used in earlier studies to characterise the motor response to intraluminal pressure changes [12-14]. However, these studies did not mimic the dynamic process of recurrent upper airway closure and reopening characterising OSA patients. Indeed, the upper airway was kept open and a progressive intratracheal negative pressure was applied to promote the reduction of the inspiratory flow (flow limitation) but without reaching full airway collapse $[12,13]$. A similar approach has recently been used to study the intraluminal volume changes in response to negative and positive pressures by means of magnetic resonance imaging [15]. Given that this technique demands the continuous application of positive or negative pressure over an extended period of time for image acquisition, the fastcycling dynamics characterising the upper airway events in OSA could not be mimicked. In another study, a quasi-static

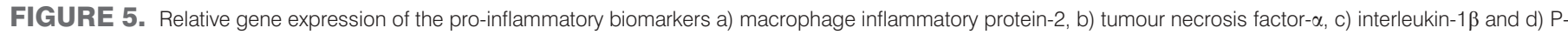

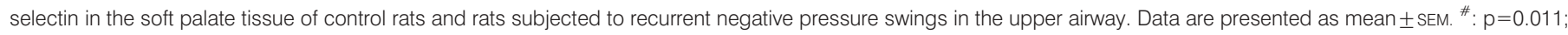
$\star * *$ : $p<0.001$.

c)

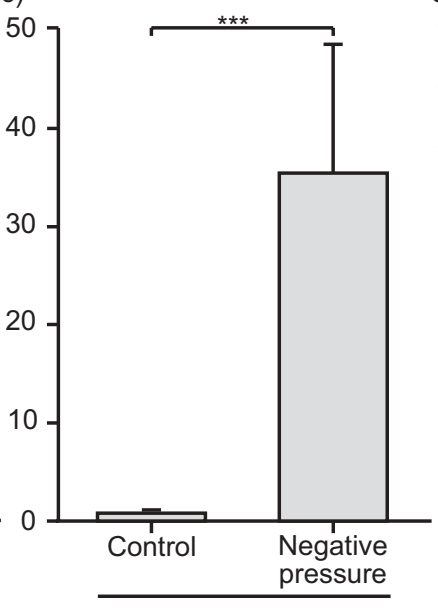

IL-1 $\beta$ d)

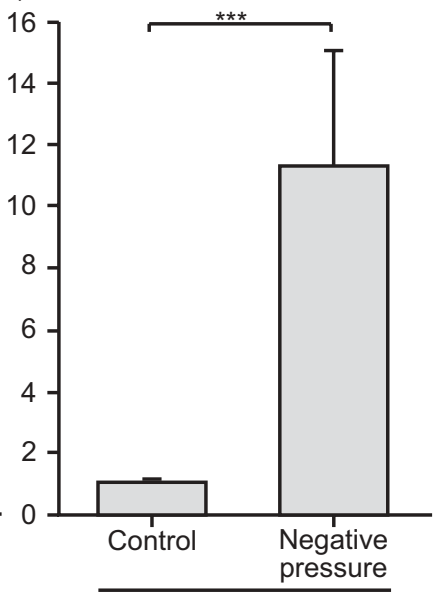

P-selectin 
negative pressure was simultaneously applied to the inlet and the outlet of the upper airway with the result that airway closure could not be documented [14]. In contrast, the model used in the present work was able to simulate the mechanical stress experienced in OSA, since the sequence of mechanical events applied to the upper airway was induced by a dynamic pattern mimicking breathing. Another advantage of this experimental setting was that it allowed the direct measurement of $P_{\text {crit }}$ (intraluminal pressure causing closure) of the upper airway instead of extrapolating data from partial collapse conditions using a Starling-resistor model, or by introducing catheters into the upper airway $[12,13,15]$.

It has recently been reported that other mechanical stimuli indirectly associated with OSA can induce an early inflammatory response in the upper airway. The application of a vibration akin to snoring triggers a pro-inflammatory cascade in human airway epithelial cells [16] and in the rat soft palate [10]. Moreover, it has been shown that the mechanical compression associated with continuous positive airway pressure can promote inflammation in the nasal mucosa of rats [17]. However, there is no direct evidence that the recurrent mechanical stress consisting of negative pressure, collapse and reopening, experienced by the upper airway, induces local inflammation. The only available data on a similar mechanical stress refer to bronchial and lung tissues. In particular, it was reported that collapse and reopening in the lower airways caused by mechanical ventilation in animal models [18] and in cultured foetal rat pulmonary epithelial cells [19] induced inflammation and cell damage, respectively. In addition, some earlier data supported the notion that negative pressure could be an inflammatory stimulus in the bronchial wall. Indeed, a recent study carried out in OSA patients showed a correlation between the levels of IL-8 in sputum and the apnoea/hypopnoea index [20]. The bronchial inflammation observed in these patients could be triggered by the increased negative pressure exerted on the bronchial tree during the recurrent inspirations with an increased upper airway resistance.

The present work provides evidence that the mechanical stress exerted by recurrent intraluminal negative pressure, collapse and reopening induces an early pro-inflammatory cascade in the upper airway. Specifically, a dramatic gene overexpression of the inflammatory biomarkers MIP-2, TNF- $\alpha$, IL-1 $\beta$ and Pselectin was observed in the larynx and soft palate tissues (figs 4 and 5, respectively). The cytokine TNF- $\alpha$ causes endothelial dysfunction [21] and regulates the expression of reactive oxygen species and adhesion molecules [22], whereas the cytokine IL-1 $\beta$ can induce the expression of other inflammatory molecules, such as MIP-2, which lead to leukocyte recruitment [23]. The adhesion molecule P-selectin, which is expressed in the endothelium, plays an important role in leukocyte extravasation through the endothelial barrier [24]. Given the complexity and redundancy of the signalling pathways of these inflammatory mediators, the interpretation of changes in the gene expression for each individual marker should be treated with caution [25]. However, the considerable overexpression observed in all the biomarkers analysed (figs 4 and 5) clearly shows that the acute stimulus applied triggered early local upregulation of the inflammatory cascade.
The inflammatory response induced by the mechanical stress in the upper airway may result in structural and functional consequences; thickening of the pharyngeal wall, local myopathy and/or neuropathy. Indeed, earlier studies have shown that the presence of cytokines such as TNF- $\alpha$ [26] and IL-1 [27] can cause weakness and contractile dysfunction of muscle fibres. Moreover, TNF- $\alpha$ could cause a loss of sensitivity in the upper airway by inducing neuropathy [28] and neurotoxicity [29].

In conclusion, the present study shows that the mechanical stress associated with recurrent intraluminal pressure swings, collapse and reopening triggers an inflammatory process in the upper airway. Accordingly, it is expected that the chronic application of this mechanical challenge can give rise to inflammatory cellular infiltration and tissue changes causing the structural and functional upper airway injuries observed in patients, contributing to the "vicious circle" that characterises the progression of obstructive sleep apnoea [30].

\section{ACKNOWLEDGEMENTS}

The authors wish to thank M.A. Rodríguez (Facultat de Medicina, Universitat de Barcelona, Barcelona, Spain) for his technical assistance.

\section{REFERENCES}

1 Farré R, Rotger M, Montserrat JM, Calero G, Navajas D. Collapsible upper airway segment to study the obstructive sleep apnea/hypopnea syndrome in rats. Respir Physiol Neurobiol 2003; 136: 199-209.

2 Calero G, Farré R, Ballester E, et al. Physiological consequences of prolonged periods of flow limitation in patients with sleep apnea hypopnea syndrome. Respir Med 2006; 100: 813-817.

3 Sekosan M, Zakkar M, Wenig BL, Olopade CO, Rubinstein I. Inflammation in the uvula mucosa of patients with obstructive sleep apnea. Laryngoscope 1996; 106: 1018-1020.

4 Anastassov GE, Trieger N. Edema in the upper airway in patients with obstructive sleep apnea syndrome. Oral Surg Oral Med Oral Pathol Oral Radiol Endod 1998; 86: 644-647.

5 Boyd JH, Petrof BJ, Hamid Q, Fraser R, Kimoff RJ. Upper airway muscle inflammation and denervation changes in obstructive sleep apnea. Am J Respir Crit Care Med 2004; 170: 541-546.

6 Kimoff RJ, Sforza E, Champagne V, Ofiara L, Gendron D. Upper airway sensation in snoring and obstructive sleep apnea. Am J Respir Crit Care Med 2001; 164: 250-255.

7 Larsson H, Carlsson-Nordlander B, Lindblad LE, Norbeck O, Svanborg E. Temperature thresholds in the oropharynx of patients with obstructive sleep apnea syndrome. Am Rev Respir Dis 1992; 146: 1246-1249.

8 Nguyen AT, Jobin V, Payne R, Beauregard J, Naor N, Kimoff RJ. Laryngeal and velopharyngeal sensory impairment in obstructive sleep apnea. Sleep 2005; 28: 585-593.

9 Ryan CM, Bradley TD. Pathogenesis of obstructive sleep apnea. J Appl Physiol 2005; 99: 2440-2450.

10 Almendros I, Acerbi I, Puig F, Montserrat JM, Navajas D, Farré R. Upper-airway inflammation triggered by vibration in a rat model of snoring. Sleep 2007; 30: 225-227. 
11 Livak KJ, Schmittgen TD. Analysis of relative gene expression data using real-time quantitative PCR and the $2^{-\triangle \Delta C T}$ method. Methods 2001; 25: 402-408.

12 Fuller DD, Williams JS, Janssen PL, Fregosi RF. Effect of coactivation of tongue protrudor and retractor muscles on tongue movements and pharyngeal airflow mechanics in the rat. J Physiol 1999; 519: 601-613.

13 Rowley JA, Williams BC, Smith PL, Schwartz AR. Neuromuscular activity and upper airway collapsibility. Mechanisms of action in the decerebrate cat. Am J Respir Crit Care Med 1997; 156: 515-521.

14 Ryan S, McNicholas WT, O'Regan RG, Nolan P. Upper airway muscle paralysis reduces reflex upper airway motor response to negative transmural pressure in rat. $J$ Appl Physiol 2003; 94: 1307-1316.

15 Van Zutphen C, Janssen P, Hassan M, Cabrera R, Bailey EF, Fregosi RF. Regional velopharyngeal compliance in the rat: influence of tongue muscle contraction. NMR Biomed 2007; 20: 682-691.

16 Puig F, Rico F, Almendros I, Montserrat JM, Navajas D, Farré R. Vibration enhances interleukin-8 release in a cell model of snoring-induced airway inflammation. Sleep 2005; 28: 1312-1316.

17 Almendros I, Acerbi I, Vilaseca I, Montserrat JM, Navajas D, Farré R. Continuous positive airway pressure (CPAP) induces early inflammation. Sleep 2008; 31: 127-131.

18 Cheng KC, Zhang H, Lin CY, Slutsky AS. Ventilation with negative airway pressure induces a cytokine response in isolated mouse lung. Anesth Analg 2002; 94: 1577-1582.

19 Bilek AM, Dee KC, Gaver DP 3rd. Mechanisms of surfacetension-induced epithelial cell damage in a model of pulmonary airway reopening. J Appl Physiol 2003; 94: 770-783.

20 Devouassoux G, Lévy P, Rossini E, et al. Sleep apnea is associated with bronchial inflammation and continuous positive airway pressure-induced airway hyperresponsiveness. J Allergy Clin Immunol 2007; 119: 597-603.

21 Gao X, Xu X, Belmadani S, et al. TNF- $\alpha$ contributes to endothelial dysfunction by upregulating arginase in ischemia/reperfusion injury. Arterioscler Thromb Vasc Biol 2007; 27: 1269-1275.

22 Mukhopadhyay S, Hoidal JR, Mukherjee TK. Role of TNF- $\alpha$ in pulmonary pathophysiology. Respir Res 2006; 7: 125.

23 Xu WB, Haddad EB, Tsukagoshi H, Adcock I, Barnes PJ, Chung KF. Induction of macrophage inflammatory protein 2 gene expression by interleukin- $1 \beta$ in rat lung. Thorax 1995; 50: 1136-1140.

24 Luscinskas FW, Ding H, Lichtman AH. P-selectin and vascular cell adhesion molecule 1 mediate rolling and arrest, respectively, of CD4+ T lymphocytes on tumor necrosis factor- $\alpha$-activated vascular endothelium under flow. J Exp Med 1995; 181: 1179-1186.

25 Elias JA, Freundlich B, Kern JA, Rosenbloom J. Cytokine networks in the regulation of inflammation and fibrosis in the lung. Chest 1990; 97: 1439-1445.

26 Reid MB, Lännergren J, Westerblad H. Respiratory and limb muscle weakness induced by tumor necrosis factor- $\alpha$ : involvement of muscle myofilaments. Am J Respir Crit Care Med 2002; 166: 479-484.

27 Salomonsson S, Lundberg IE. Cytokines in idiopathic inflammatory myopathies. Autoimmunity 2006; 39: 177-190.

28 Créange A, Lefaucheur JP, Authier FJ, Gherardi RK. [Cytokines and peripheral neuropathies]. Rev Neurol (Paris) 1998; 154: 208-216.

29 Nguyen HX, O’Barr TJ, Anderson AJ. Polymorphonuclear leukocytes promote neurotoxicity through release of matrix metalloproteinases, reactive oxygen species, and TNF- $\alpha$. J Neurochem 2007; 102: 900-912.

30 Friberg D. Heavy snorer's disease: a progressive local neuropathy. Acta Otolaryngol 1999; 119: 925-933. 